\title{
Enhancement of low-energy electron emission in 2-D radioactive films
}

\author{
Alex Pronschinske ${ }^{1}$, Philipp Pedevilla ${ }^{2}$, Colin J. Murphy ${ }^{1}$, Emily A. Lewis ${ }^{1}$, Felicia R. Lucci ${ }^{1}$, Garth Brown ${ }^{3}$, \\ George Pappas ${ }^{3}$, Angelos Michaelides ${ }^{2} \&$ E. Charles H. Sykes ${ }^{1}$
}

\begin{abstract}
High-energy radiation has been utilized for decades, however, the role of low-energy electrons created during irradiation has only recently begun to be appreciated ${ }^{1,2}$. Low-energy electrons are the most important component of radiation damage in biological environments because they have subcellular ranges, interact destructively with chemical bonds, and are the most abundant product of ionizing particles in tissue. However, methods for generating them locally without external stimulation do not exist. To address this we synthesized one atom thick films of the radioactive isotope ${ }^{125} \mathrm{I}$ on gold that are stable under ambient conditions. Scanning tunnelling microscopy, supported by electronic structure simulations, allowed us to directly observe nuclear transmutation of individual ${ }^{125} \mathrm{I}$ atoms into ${ }^{125} \mathrm{Te}$, and explain the surprising stability of the 2-D film as it underwent radioactive decay. The metal interface geometry induces a $600 \%$ amplification of low-energy electron emission $(<10 \mathrm{eV})^{3}$ compared to atomic ${ }^{125}$ I. Therefore, this enhancement of biologically active low energy electrons opens a new direction for highly targeted nanoparticle therapies ${ }^{4-6}$.
\end{abstract}

Nuclear decay is one of the most extreme processes and is central to a range of fields including energy, medicine, imaging, labelling, archaeology and sensing. Radiation in the form of alpha particles, beta particles and gamma rays have fundamentally different interactions with matter and therefore exhibit different mean-free paths ( $\sim 1 \mu \mathrm{m}, 1 \mathrm{~mm}$ and $1 \mathrm{~cm}$, respectively). These forms of primary radiation deposit their energy over the course of their trajectory by ionizing their surroundings and producing non-thermal secondary electrons. Only very recently has the ability of low-energy secondary electrons to induce chemical reactions and biological damage begun to be appreciated $^{1}$, because they have energies below the typical ionization threshold of organic matter. For example, low-energy electrons (3-20 eV) have been shown to be effective at causing DNA cleavage ${ }^{2,4,7}$. This ability stems from their high cross-section for breaking chemical bonds, and as a consequence they have a very short meanfree path of $\sim 1-10 \mathrm{~nm}$ in solution ${ }^{8,9}$. Furthermore, hot electrons that are not captured by surrounding molecules become thermalized as solvated electrons which are known to be chemically and biological active ${ }^{9-12}$. To harness these unique properties, the design of radioactive materials that increase and localize the flux of short-range lowenergy electrons to target sites is crucial for their application in targeted cancer therapies that minimize damage to healthy cells. Thus far, it has not been possible to design atomically precise radioactive materials that maximize these effects due to self-destruction arising from nuclear recoil, Coulomb explosion and self-irradiation ${ }^{13-16}$.

We report a straightforward method for synthesizing monolayer films of radioactive ${ }^{125} \mathrm{I}$ atoms on gold-coated mica substrates under ambient conditions, and characterize their composition and their electron emission. Despite being synthesized from radioactive ${ }^{125} \mathrm{I}$ ( $>99.9 \%$ purity) they are robust with respect to self-destruction, and provide well-defined, intense planar sources of secondary electrons. ${ }^{125} \mathrm{I}$ decays by electron capture (EC) of a core shell electron to produce a nuclear excited state of ${ }^{125} \mathrm{Te}$ (Figure 1a), the majority of which eject another core 
shell electron during de-excitation. A cascade of electronic relaxations following the creation of each core hole leads to emission of multiple electrons. Most of these emitted electrons have $>10 \mathrm{eV}$ kinetic energy and their distribution in energy is very sensitive to the local chemical environment ${ }^{3,15,17}$. This rapid electron emission (within $\sim 1 \mathrm{~ns}$ ) leaves the daughter ${ }^{125} \mathrm{Te}$ atom in a highly charged state (up to $\mathrm{Te}^{25+}$ ), and in a condensed material or molecule the sudden charging makes the system susceptible to fragmentation (via Coulomb explosion) without sufficiently fast neutralization ${ }^{15}$. Therefore, a major challenge in realizing a nano-structured radiation source is the design of a system that is robust under the ultra-fast release of energy and particles that accompany each atomic decay event. In anticipation of this challenge we have chosen ${ }^{125} \mathrm{I} / \mathrm{Au}$ for this work, because the wellknown, robust I/Au chemistry makes this system a good candidate for a stable 2-D emitter ${ }^{18,19}$.

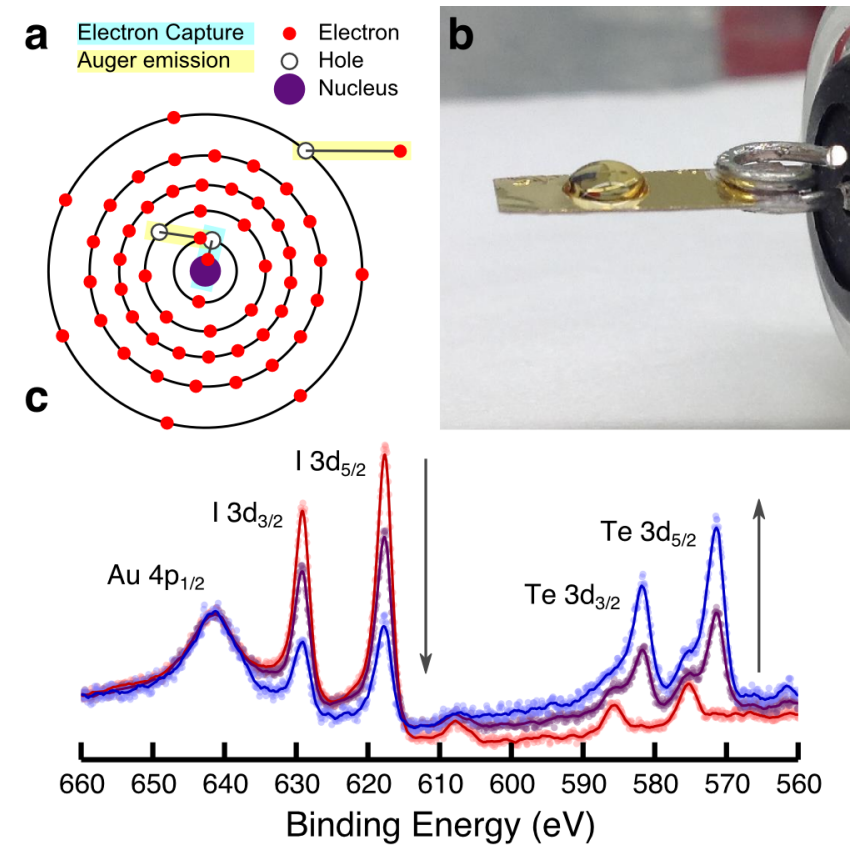

\begin{tabular}{l|l} 
Figure & Overview of radioactive ${ }^{125} I$ monolayer \\
film preparation and characterization.
\end{tabular} (a) Schematic of electron capture decay of ${ }^{125} \mathrm{I}$ and a resulting Auger electron. (b) Photograph of Au/mica substrate during the drop-casting deposition of I from solution. (c) XPS spectra of radioactive ${ }^{125}$ I film as a function of time reveals the nuclear transmutation of ${ }^{125} \mathrm{I}$ into ${ }^{125} \mathrm{Te}$, and indicates that the daughter ${ }^{125} \mathrm{Te}$ atom remains bound to the surface. Elemental core levels are labelled and arrows highlight the change of I to Te over time: red is at 8 days, purple at 38 days and blue at 76 days.

Samples were prepared using an ambient drop-casting method adapted from the previous (non-radioactive) ${ }^{127} \mathrm{I} / \mathrm{Au}$ work of Huang et al. ${ }^{20}$ (Figure 1b). Survey X-ray photoelectron spectra (XPS) taken after preparation and transportation indicate no major contamination of the ${ }^{125} \mathrm{I}$ samples during deposition or exposure to ambient conditions (Figure S1). In order to track the nuclear transmutation of ${ }^{125} \mathrm{I}$ to ${ }^{125} \mathrm{Te}$, XPS measurements of the sample were taken as a function of time. By measuring the I and Te 3d core levels with XPS we directly observe the nuclear transmutation of ${ }^{125} \mathrm{I}$ to ${ }^{125} \mathrm{Te}$ as a decrease in the I and an increase in the Te signals over time (Figure 1c). The sample never left vacuum over the course of the XPS measurements and hence the newly formed ${ }^{125} \mathrm{Te}$ atoms appear in the spectrum with a binding energy of $582\left(3 \mathrm{~d}_{3 / 2}\right)$ and $572\left(3 \mathrm{~d}_{5 / 2}\right) \mathrm{eV}$, as expected for the $\mathrm{Te}^{0}$ oxidation state ${ }^{21}$. These XPS measurements clearly show that the films withstand ambient processing (based on initial XPS surveys after synthesis and transportation), and that the newly formed ${ }^{125} \mathrm{Te}$ daughter is resistant to desorption. 

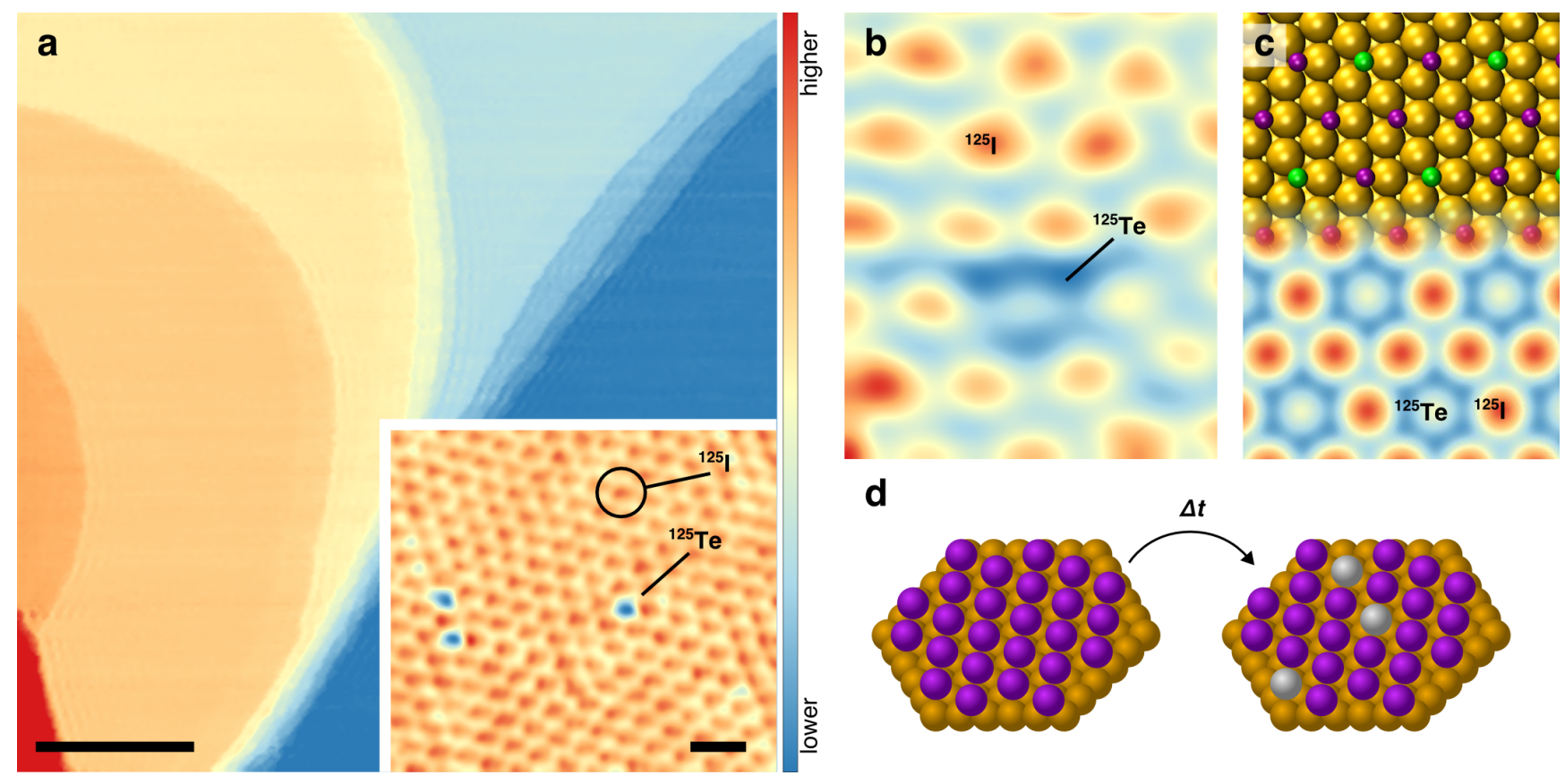

Figure | Atomic-scale characterization of radioactive ${ }^{125}$ I monolayer and nuclear transmutation. (a) STM images of ${ }^{125} \mathrm{I}$ films show flat I-covered Au terraces separated by atomic steps (main image: $\mathrm{V}=+100 \mathrm{mV}, \mathrm{I}=1$ $\mathrm{nA}$ and $50 \mathrm{~nm}$ scale bar) and atomic resolution of ${ }^{125} \mathrm{I}$ atoms in $(\sqrt{3} \times \sqrt{ } 3) \mathrm{R} 30^{\circ}$ overlayer (inset: $\mathrm{V}=-400 \mathrm{mV}, \mathrm{I}=$ $100 \mathrm{pA}$ and $1 \mathrm{~nm}$ scale bar). (b) High resolution STM image reveals a ${ }^{125} \mathrm{Te}$ atom feature topographically lower than the neighbouring I atoms (V = $100 \mathrm{mV}, \mathrm{I}=10 \mathrm{pA})$. (c) Repeating DFT structure (top; I in purple and Te in green) and DFT-based simulated STM image (bottom) indicate that these features are isolated Te atoms which appear topographically lower than their I neighbours due to the shorter, stronger Te-Au bond. (d) Schematic representation of stochastic nuclear transmutation of individual atoms in the monolayer geometry $\left({ }^{125} \mathrm{I}\right.$ in purple, ${ }^{125} \mathrm{Te}$ in grey).

We imaged the film structure with scanning tunnelling microscopy (STM) to search for atomic-scale damage near the ${ }^{125}$ Te species observed in XPS. Theoretical studies have indicated that the decay of condensed phase ${ }^{125} \mathrm{I}$ leads to an average total energy of $18.3 \mathrm{keV}$ being deposited into its surroundings in the form of hot electrons ${ }^{3,22}$. When using the traditional convention of only considering total deposited energy, it would be reasonable to suspect film damage via local atomic desorption in ${ }^{125} \mathrm{I}$ films ${ }^{18}$. However this is not what we observe; when imaging $100 \times 100 \mathrm{~nm}^{2}$ areas or larger (Figure Figure a), no damage is visible, and the ${ }^{125}$ I films appear identical to those of stable ${ }^{127}$ I control films (Figure S2). High-resolution imaging of smaller areas (Figure Figure a, inset; and Figure $2 b)$ yields atomic resolution of the ${ }^{125} \mathrm{I}$ monolayer in the expected $(\sqrt{ } 3 \times \sqrt{ } 3) \mathrm{R} 30^{\circ}$ structure. In total, imaging of the radioactive monolayer structure at many scales shows that the ${ }^{125} \mathrm{I}$ film is not damaged by selfirradiation. We observe the appearance of atom-sized depressions randomly distributed throughout the ${ }^{125} \mathrm{I}$ monolayer which are not present in the ${ }^{127}$ I control films which we assign as ${ }^{125} \mathrm{Te}$ atoms resulting from the nuclear transmutation of ${ }^{125} \mathrm{I}$. 
a

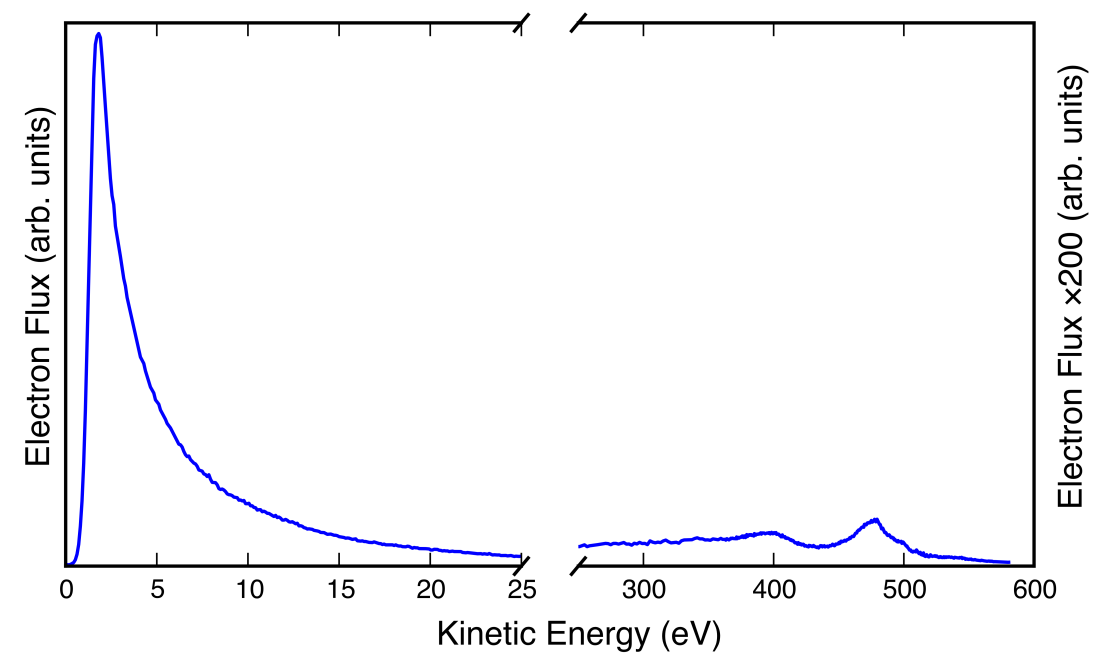

b

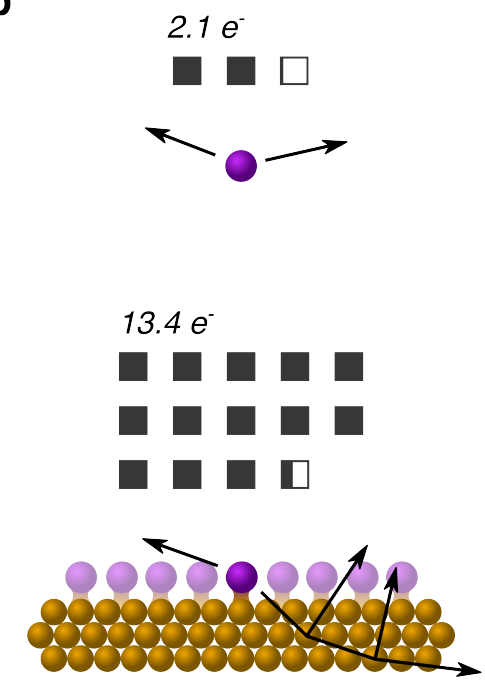

Figure | Electron emission from radioactive ${ }^{125}$ I monolayer. (a) Representative electron emission spectrum shows that, in addition to the expected electron capture decay process Auger peaks at $480 \mathrm{eV}$ (intensity $\times 200$ ), a multitude of low-energy $(0-20 \mathrm{eV})$ electrons are emitted from the ${ }^{125} \mathrm{I} / \mathrm{Au}$ film. (b) Schematic of electron backscattering from the metal film (lower) which leads to six-fold enhancement of low-energy electron emission compared to atomic ${ }^{125} \mathrm{I}$ (upper). Each box represents the average emission of 1 electron per decay with kinetic energy $<10 \mathrm{eV}^{3}$.

The striking resilience of the radioactive film can be explained by the density functional theory calculations (DFT) we performed for various I, Te and mixed I/Te overlayers. We determined the most stable adsorption sites for both I and Te as the three-fold hollow sites by placing the adsorbates at a variety of high-symmetry adsorption sites (for an explanation of DFT methods and setup see Supplementary Information). The simulated STM images of the overlayer structure (illustrated in Figure Figure c) indicate that the Te atoms do indeed appear as depressions with respect to the I atoms (See Figures S3 \& S4 where it is shown that this is the case for all biases considered). This difference in apparent height is primarily due to the Te atoms being bound more strongly to the $\mathrm{Au}(111)$ surface and subsequently having a shorter bond length (268 pm for Te, 291 pm for I). Experimental images with STM tip states that provide very high spatial resolution reveal a central protrusion within the depressions corresponding to the Te atom, which is consistent with our DFT-simulated STM images (Figure 2b, c). Furthermore, the results of the DFT calculations explain the experimentally observed film stability. The calculated I-Au and Te-Au binding energies, $2.1 \mathrm{eV}$ and $3.1 \mathrm{eV}$ respectively, are much larger than the nuclear recoil energy $(<0.1 \mathrm{eV})$, thus preventing rupture of the surface bonds following decay ${ }^{18}$. More importantly, there is also substantial hybridization of the I/Te valence orbitals with the Au surface which we postulate imparts resilience against Coulomb explosion by allowing fast electron transfer from the Au surface that rapidly neutralizes the atom undergoing decay ${ }^{15,23}$.

In order to probe the performance of the ${ }^{125} \mathrm{I} / \mathrm{Au}$ films as low-energy electron emitters we recorded electron emission spectra in the 0-600 eV kinetic energy range with a 5-channel concentric hemispherical electron energy 
analyser. The emission spectrum (Figure 3a) shows the electron energy distribution is concentrated in the desired region of $0-20 \mathrm{eV}$ and a smaller peak at $480 \mathrm{eV}$ that arises from Auger (MNN) transitions in the daughter ${ }^{125} \mathrm{Te}$ atom during the core hole relaxation cascade ${ }^{21}$. Given the uncertainty of the transmission function of our analyser at low electron energy we quantified the number of emitted electrons by bringing a flat Au-coated plate (3.5 mm diameter) within $0.1 \mathrm{~mm}$ of the grounded sample surface in vacuum and recording the electrical current. By applying a negative potential to the collector plate we could suppress, and hence quantify, the low-energy electron flux flowing from the radioactive sample to the collector. Application of $-10 \mathrm{~V}$ to the collector plate suppressed the electron flow by $11.8 \mathrm{pA}$. The half-life of ${ }^{125} \mathrm{I}$, the age of our sample (8 days), and the density of ${ }^{125} \mathrm{I}$ atoms in the monolayer are known, and we calculate that there are 13.4 electrons emitted per ${ }^{125}$ I decay with a kinetic energy $<10 \mathrm{eV}$ (see Supplementary Information for details). The 2-D interface geometry of our radiation source dictates that half of the primary electrons emitted from decaying ${ }^{125} \mathrm{I}$ atoms are directed into the metal substrate, and those with higher energies should be expected to backscatter lower energy secondary electrons; in an analogous manner to secondaries generated during electron spectroscopy and diffraction experiments ${ }^{24}$. An interesting effect occurs when the collector is biased at $-10 \mathrm{~V}$; the net current flow reverses compared to when the sample and collector are unbiased, and (at $-10 \mathrm{~V}$ ) electrons flow from the collector to the sample. This is because the high energy primaries from the radioactive sample generate secondary electrons in the collector plate that dominate the electrical current when the secondary electrons from the sample are suppressed. Although we expect some enhancement of the low-energy primary electron emission due to hybridization between the ${ }^{125} \mathrm{I}$ atoms and the Au surface, this experiment demonstrates the significance of the secondary emission from Au metal induced by primary emission of the radioactive decay. Our ${ }^{125} \mathrm{I}$ films emit a low-energy $(0-10 \mathrm{eV})$ electron flux that is $>600 \%$ that of atomic ${ }^{125} \mathrm{I}$ when deposited on $\mathrm{Au}(111$ ) (Figure $3 \mathrm{~b}$ ) mainly via inelastic scattering, which will be a ubiquitous property for all metal substrates. Furthermore, the influence of the substrate material can be examined using this setup. We found a $\sim 20 \%$ reduction in the secondary emission induced by the ${ }^{125}$ I monolayer in a $\sim 0.6$ $\mathrm{mm}$ thick disc of graphite attached to the collector, which illustrates the benefits of a metallic substrate in amplifying the yield of chemically-active low-energy electrons.

In summary, we report a method for making air-stable, planar and atomically well-defined radiation sources that emit high fluxes of low-energy electrons. This system has enabled us to image nuclear transmutation with atomic-scale resolution. Looking forward, the simplicity of this approach for making 2-D radioactive films, coupled with the abundance of established experimental procedures for nano-patterning substrates and functionalizing nanoparticles will open up many new possibilities. For example, electron transmission through thin films of water, proteins and DNA adsorbed on well-defined radioactive substrates will help quantify the local effect of secondary electrons. This will in turn provide an improved microscopic understanding of radiation chemistry, biological degradation and material damage ${ }^{25}$. In terms of application, ${ }^{125}$ I is commonly used in radiation therapy as well as in medical imaging and the I-Au surface chemistry used in our model system is compatible with Au nanoparticles. Such nanoparticles, when functionalized, are commonly used in many aspects of biology and medicine to target specific sites within cells ${ }^{5,6,26-29}$. Given that $80 \%$ of the secondary-generating electrons ejected from ${ }^{125} \mathrm{I}$ have an inelastic mean free path less than $2 \mathrm{~nm},{ }^{125} \mathrm{I}$-coated nanoparticles are expected to also produce high fluxes of low-energy electrons. The prospects for ${ }^{125} \mathrm{I} / \mathrm{Au}$ nanoparticle stability in a biological environment are promising as it is well known that iodine-coated Au nanoparticles are very stable in solution due 
to strong I-Au bonds, and our DFT calculations reveal that the Te-Au bond is even stronger ${ }^{6,30-32}$. The $600 \%$ amplification in low-energy electron emission of the radioactive ${ }^{125} \mathrm{I} / \mathrm{Au}$ system we report here highlights the potential for targeted radio-iodine coated Au nanoparticles that increase their efficacy per nuclear decay while minimizing bystander damage due to the short mean free path of the emitted low-energy electrons.

1. Arumainayagam, C. R., Lee, H.-L., Nelson, R. B., Haines, D. R. \& Gunawardane, R. P. Low-energy electron-induced reactions in condensed matter. Surf. Sci. Rep. 65, 1-44 (2010).

2. Boudaïffa, B., Cloutier, P., Hunting, D., Huels, M. a \& Sanche, L. Resonant formation of DNA strand breaks by low-energy (3 to $20 \mathrm{eV}$ ) electrons. Science 287, 1658-1660 (2000).

3. Pomplun, E., Booz, J. \& Charlton, D. E. A Monte Carlo simulation of Auger cascades. Radiat. Res. 111, 533-552 (1987).

4. Balagurumoorthy, P., Xu, X., Wang, K., Adelstein, S. J. \& Kassis, A. I. Effect of distance between decaying 125I and DNA on Auger-electron induced double-strand break yield. Int. J. Radiat. Biol. 88, 998-1008 (2012).

5. McLaughlin, M. F. et al. Gold coated lanthanide phosphate nanoparticles for targeted alpha generator radiotherapy. PLoS One 8, e54531 (2013).

6. Kim, Y.-H. et al. Tumor targeting and imaging using cyclic RGD-PEGylated gold nanoparticle probes with directly conjugated iodine-125. Small 7, 2052-60 (2011).

7. Alizadeh, E., Sanz, A. G., Garcia, G. \& Sanche, L. Radiation Damage to DNA: The Indirect Effect of Low-Energy Electrons. J. Phys. Chem. Lett. 4, 820-825 (2013).

8. Pimblott, S. \& LaVerne, J. Production of low-energy electrons by ionizing radiation. Radiat. Phys. Chem. 76, 1244-1247 (2007).

9. Siefermann, K. R. et al. Binding energies, lifetimes and implications of bulk and interface solvated electrons in water. Nat. Chem. 2, 274-9 (2010).

10. Garrett, B. C. et al. Role of water in electron-initiated processes and radical chemistry: issues and scientific advances. Chem. Rev. 105, 355-389 (2005).

11. Alexander, W. A., Wiens, J. P., Minton, T. K. \& Nathanson, G. M. Reactions of solvated electrons initiated by sodium atom ionization at the vacuum-liquid interface. Science 335, 1072-5 (2012).

12. Alizadeh, E. \& Sanche, L. Precursors of solvated electrons in radiobiological physics and chemistry. Chem. Rev. 112, 5578-602 (2012).

13. Chen, S. P., Hawley, M., Van Stockum, P. B., Manoharan, H. C. \& Bauer, E. D. Surface structure of cleaved (001) USb2 single crystal. Philos. Mag. 89, 1881-1891 (2009).

14. Zhu, L. et al. Neutrino-recoil induced desorption. J. Vac. Sci. Technol. A Vacuum, Surfaces, Film. 12, 2037-2044 (1994).

15. Verkhoturov, S. et al. Auger Stimulated Ion Desorption of Negative Ions via K-Capture Radioactive Decay. Phys. Rev. Lett. 87, 37601 (2001).

16. Booth, C. et al. Quantifying structural damage from self-irradiation in a plutonium superconductor. Phys. Rev. B 76, 064530 (2007).

17. Gokhberg, K., Kolorenč, P., Kuleff, A. I. \& Cederbaum, L. S. Site- and energy-selective slow-electron production through intermolecular Coulombic decay. Nature 505, 661-3 (2014).

18. Ertl, H. H., Feinendegen, L. E. \& Heiniger, Hj. J. Iodine-125, a tracer in cell biology: physical properties and biological aspects. Phys. Med. Biol. 15, 447 (1970).

19. Yamada, T., Batina, N. \& Itaya, K. Structure of electrochemically deposited iodine adlayer on Au(111) studied by ultrahighvacuum instrumentation and in situ STM. J. Phys. Chem. 99, 8817-8823 (1995).

20. Huang, L., Zeppenfeld, P., Horch, S. \& Comsa, G. Determination of iodine adlayer structures on Au(111) by scanning tunneling microscopy. J. Chem. Phys. 107, 585 (1997).

21. Chastain, J. \& Moulder, J. F. Handbook of X-ray Photoelectron Spectroscopy: A Reference Book of Standard Spectra for Identification and Interpretation of XPS Data. (Physical Electronics, 1995).

22. Katakura, J., Oshima, M., Kitao, K. \& Iimura, H. Nuclear data sheets for A=125. Nucl. Data Sheets 70, 217-314 (1993). 
23. Nath, A., Prushan, M. J. \& Gilbert, J. G. Can super-excited molecules survive fragmentation? J. Radioanal. Nucl. Chem. 247, 589-591 (2001).

24. Kolasinski, K. W. Surface Science: Foundations of Catalysis and Nanoscience. (Wiley, 2012).

25. Brun, É., Cloutier, P., Sicard-Roselli, C., Fromm, M. \& Sanche, L. Damage induced to DNA by low-energy (0-30 eV) electrons under vacuum and atmospheric conditions. J. Phys. Chem. B 113, 10008-10013 (2009).

26. Setua, S., Ouberai, M., Piccirillo, S. G., Watts, C. \& Welland, M. Cisplatin-tethered gold nanospheres for multimodal chemoradiotherapy of glioblastoma. Nanoscale 6, 10865-10873 (2014).

27. Dam, D. H. M. et al. Direct observation of nanoparticle-cancer cell nucleus interactions. ACS Nano 6, 3318-3326 (2012).

28. Ghosh, P., Han, G., De, M., Kim, C. K. \& Rotello, V. M. Gold nanoparticles in delivery applications. Adv. Drug Deliv. Rev. 60, 1307-15 (2008).

29. Bardhan, R., Lal, S., Joshi, A. \& Halas, N. Theranostic nanoshells: from probe design to imaging and treatment of cancer. Acc. Chem. Res. 44, 936-946 (2011).

30. Cochran, S. A. \& Farrell, H. H. The chemisorption of iodine on gold. Surf. Sci. 95, 359-366 (1980).

31. Cheng, W., Dong, S. \& Wang, E. Iodine-induced gold-nanoparticle fusion/fragmentation/aggregation and iodine-linked nanostructured assemblies on a glass substrate. Angew. Chemie - Int. Ed. 42, 449-452 (2003).

32. Magnussen, O. M. Ordered Anion Adlayers on Metal Electrode Surfaces Ordered Anion Adlayers on Metal Electrode Surfaces. Chem. Rev. 102, 679-726 (2002).

Acknowledgments We thank Mitch McVey, Andrew Gellman, Elena Rybak-Akimova, Arthur Utz and Sam Thomas for advice and useful discussions. We are grateful to Geoff Sirr, Chris Rock and Harry Bernheim for their oversight of safety protocols during the experiments. The work at Tufts was supported by the National Science Foundation under grants CHE0844343/CHE-1412402 (A. P., C. J. M. and E. S.). E.L. thanks the Division of Chemical Sciences, Office of Basic Energy Sciences, Condensed Phase and Interfacial Molecular Science Program, US Department of Energy (Grant No. FG0210ER16170) for support. Some of the research at UCL leading to these results has received funding from the European Research Council under the European Union’s Seventh Framework Programme (FP/2007-2013)/ERC Grant Agreement No. 616121 (HeteroIce project) and the Royal Society through a Wolfson Research merit Award (A. M.). P. P. and A. M. are grateful for computational resources to the London Centre for Nanotechnology and the UK's national high performance computing service HECToR (from which access was obtained via the UK’s Material Chemistry Consortium, EP/F067496).

Author Contributions E.C.H.S. and A.P. conceived and designed the experiments. A.P., C.J.M. and G.B. fabricated the samples. A.P., C.J.M., E.A.L. and F.R.L. carried out STM, XPS and electron emission experiments. A.P. analysed the experimental data and wrote the paper. P.P. and A.M. performed theoretical computations. G.P. and G.B. provided materials, radiation-safe laboratory space and safety oversight. All the authors discussed the results and edited the manuscript.

Author Information Correspondence and requests for materials should be addressed to E.C.H.S. (charles.sykes@tufts.edu) 